\title{
New Trends in Protein Nanocrystallography Based On LB Nanotemplate, Cell Free Expression, SNAP APA and Montecarlo: A Review
}

\author{
Claudio Nicolini* ${ }^{*}$ and Eugenia Pechkova ${ }^{2}$ \\ ${ }^{1}$ Nanoworld Institute Fondazione E. L.B.A. Nicolini, Pradalunga (Bergamo, Italy), Italy \\ ${ }^{2}$ Laboratories of Biophysics and Nanobiotechnology, Department Experimental Medicine, University of Genova, Italy
}

\begin{abstract}
A review of the emerging trends and perspectives of Protein Nanocrystallography is here presented at the intersection of advances in nanotechnology (Langmuir-Blodgett and Anodic Porous Allumina), proteomics (microarray, cell free expression and SNAP) and synchrotron radiation (third generation sources trillion times more brilliant requiring quite smaller crystals and Montecarlo simulation). It should be noted that nanocrystallography here does not refer to crystals of nanometer size or to nanodrop crystallization technology, but to the significant applications for medicine emerging in our labs at the interface of Langmuir-Blodgett engineering, organic chemistry, molecular dynamics and label-free Protein Arrays, utilizing bacterial hell's gate globin, octapus rodhopsin, bovine cytochrome, human kinase, laccase and many other proteins.
\end{abstract}

Keywords: Protein nanocrystallography; Montecarlo simulation; Langmuir-Blodgett; Cell Free Expression; SNAP APA; Protein arrays

\section{Introduction}

It was shown recently [1] that one cannot indefinitely compensate for small crystal size with increased beam intensity since at some stage so much X-ray energy is being deposited in a small volume that the protein structure and crystalline order will be destroyed by primary radiation damage very quickly. Radiation damage to crystalline proteins using X-rays is indeed a problem, which limits the structural information that can be extracted from the sample and only the significant radiation stability induced in the crystal formed by our LB nanotemplate method [2] has opened new avenues in structural proteomics obtaining protein crystals for the most important proteins like membrane proteins with required quality, quantity (easy and speed of production) and reduced radiation damage [3]. These requirements remain the major open problems in Protein Crystallography that can be overcome by the emerging trends of my research group activities [4-23] here reviewed for the Structure-Function determination of numerous important proteins as the membrane ones still unsolved.

\section{LB Nanotemplate}

This unique method of producing high quality protein crystal based on Langmuir-Blodgett nanotemplate (Figure 1) was introduced time ago with a detailed protocol [2,3] applicable to the any protein including proteins of high molecular weight and membrane proteins [49]. Indeed, a number of protein non crystallizable by classical methods were successfully crystallized by this LB nanotemplate method. In more recent times the process itself was monitored by several sophisticated techniques such as micro- and nano GISAX [10,11] and Raman spectroscopy [12], giving the insight to the described LB-related phenomena which triggers an acceleration in protein crystallization and dramatic changes in water content [13] and radiation resistance $[9,14]$. The crystals obtained by LB method are characterized by micro [4-9] and nano [10,11] focused synchrotron radiation diffraction, Atomic Force Microscopy [15] and Raman Spectroscopy [12], and following Laser micro dissection [16] they appear still well ordered and radiation stable to an unmatched level [16], in comparison of crystal obtained by classical crystallization methods which instead in presence of the high laser exposure are destroyed and disappear after 20 minutes [16]. A protein will stay in solution only up to a certain concentration.
Once this limiting concentration is reached, the solution will no longer remain homogeneous, but a new state or phase will appear. This phenomenon forms the basis of all protein crystallization experiments. By changing the solution conditions, the crystallographer tries to exceed the solubility limit of the protein so as to produce crystals. This plan rarely runs smoothly. After changing the solution conditions, one of several difficulties is usually encountered: (i) nothing happens, i.e., the protein solution remains homogeneous; (ii) a new phase appears, but it is not a crystal. Instead, it is an aggregate or a liquid; or (iii) crystals do form, but they are unsuitable for structure determination because they give a poor X-ray diffraction pattern. It is often possible to overcome these difficulties by trial and error-repeated crystallization attempts with many different conditions - but this strategy does not always work. Even when it is successful, the lessons learned cannot be easily generalized; the conditions, which work with one protein, are not necessarily optimal for a different protein. The problems associated with producing protein crystals have stimulated fundamental research on protein crystallization. An important tool in this work is the phase diagram. A complete phase diagram shows the state of a material as a function of all of the relevant variables of the system [1]. For a protein solution, these variables are the concentration of the protein, the temperature and the characteristics of the solvent (e.g., $\mathrm{pH}$, ionic strength and the concentration and identity of the buffer and any additives). The most common form of the phase diagram for proteins is two-dimensional and usually displays the concentration of protein as a function of one parameter, with all other parameters held constant. Worthy of notice and highly significant (Figure 1) is the uniqueness of LB protein crystal being analogue to space crystal by both clustering analysis and molecular dynamics [3].

*Corresponding author: Claudio Nicolini, Nanoworld Institute Fondazione EL.B.A. Nicolini, Pradalunga (Bergamo, Italy), USA and Russia, Tel: +3901035338217; Fax: +39010 35338215; E-mail: clannicolini@gmail.com

Received August 25, 2014; Accepted October 30, 2014; Published November 06, 2014

Citation: Nicolini C, Pechkova E (2014) New Trends in Protein Nanocrystallography Based On LB Nanotemplate, Cell Free Expression, SNAP APA and Montecarlo: A Review. J Microb Biochem Technol 6: 366-369. doi:10.4172/1948-5948.1000170

Copyright: @ 2014 Nicolini C, et al. This is an open-access article distributed under the terms of the Creative Commons Attribution License, which permits unrestricted use, distribution, and reproduction in any medium, provided the original author and source are credited 

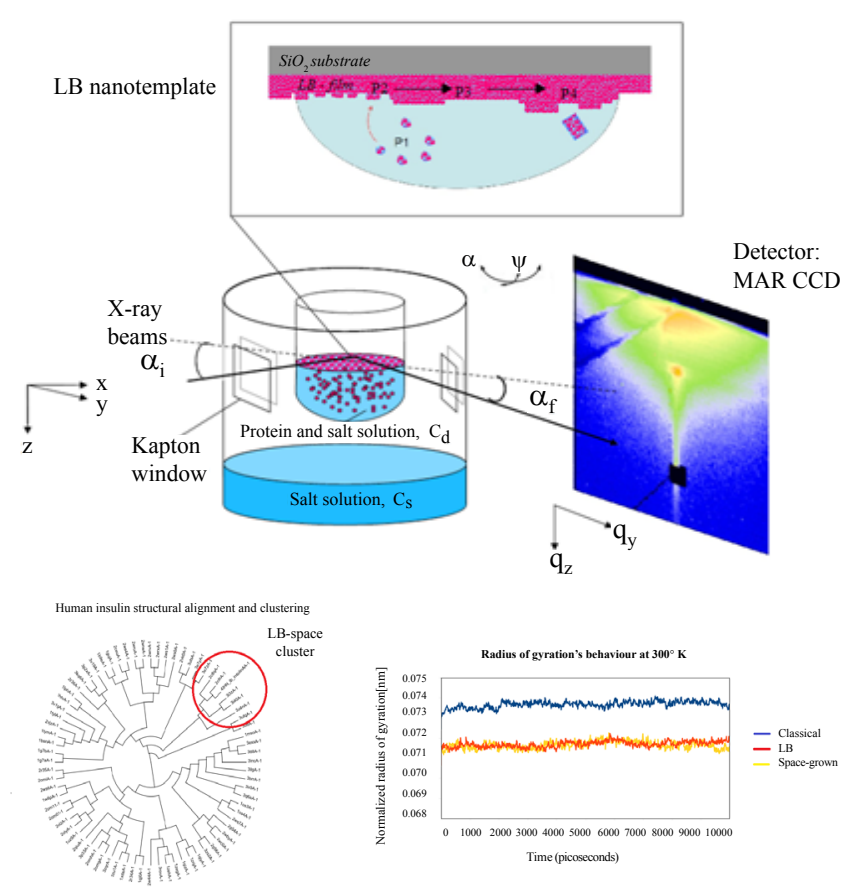

Figure 1: (left, above) Langmuir-Blodgett Nanotemplate. Uniqueness of LB protein Crystal analogue to space crystal by clustering analysis (below, left) and molecular dynamics (below, right), as shown in Reference 3.

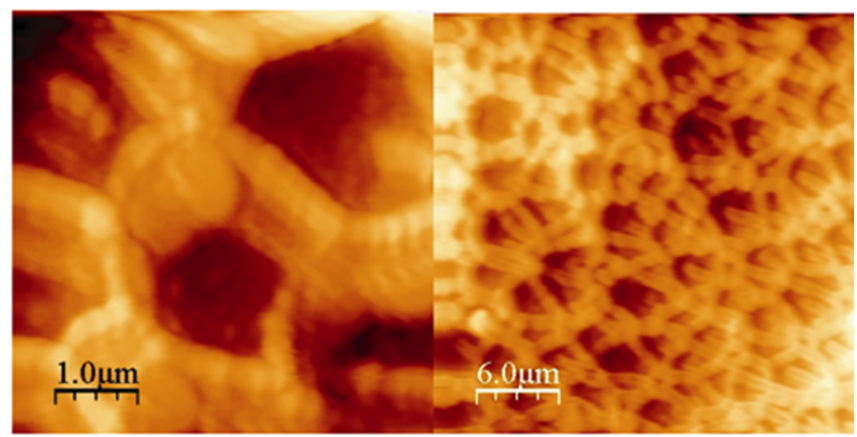

Figure 2: Morphometry of Anodic Porous Allumina microarrays by AFM as shown in Reference 17

\section{Anodic Porous Allumina}

Anodic Porous Allumina (APA) provides a structure with very thin walls [17] and its fragility must be taken into account handling the patterned surface (Figure 2, above). Two tests were carried out to characterize mechanical properties of APA grown over aluminum: a grip test (Figure 2, below) and a ball-crush test, both carried out in compliance with the DIN standards for mechanical evaluation. We have proved the reliability of the APA surface in connection with the microarray technology, including the mechanical interactions in the printing step, the adaptability to chemical treatments and to favor the protein expression within the same protocol developed for more conventional substrates as glass, mica, teflon or nylon and gold [17]. The favorable result suggests the prospect to confine the biological process including genes and antibodies within a single pore in future, improving towards smaller scales the present expression process that takes place within a statistical distribution among many nearby pores. APA faces the goal to achieve independent pore processes in arrays separated by few microns with respect to millimeter distances on glass or other flat surfaces necessary nowadays. Present work, however, has pointed out the need to overcome the hydrodynamical conditions conflicting in addressing the printed gel solution precisely at a micrometer scale [17].

\section{Cell Free Expression}

Since the early pioneering work of Nirenberg and Matthaei, which demonstrated in vitro protein translation using cell extracts, cell-free protein synthesis has become an important tool for life scientists and played a central role in a wide variety of applications. In the postgenomic era, cell-free protein synthesis is becoming one of the most important high throughput technologies for functional genomics and proteomics. The cell-free protein synthesis is the quickest way to obtain an expressed protein in vitro from a gene using PCR or plasmid template. Commercially available cell-free protein synthesis systems are typically derived from cell extracts of Escherichia coli S30, rabbit reticulocytes or wheat germ, which is like a "black box". The PURE system, Protein synthesis Using Recombinant Elements (in vitro [18], reconstitutes the $E$. coli translation machinery with fully recombinant proteins. These include 10 translation factors, 20 aminoacyl-tRNA synthetases and several enzymes for energy regeneration. In addition, recombinant T7 RNA polymerase is used to couple transcription to translation. This avoids the "black box" nature of the cell extract and has the capacity for a yield of more than $100 \mu \mathrm{g} / \mathrm{ml}$, being now exclusively licensed to New England Biolabs (Ipswich, MA, USA) under the tradename "PURExpress" [18]. The fluid exchanges on the APA arrays (mixing reagents and washing them away) appear optimal for SNAP arrays/pores [19], even if the open pore design to force fluid through the device and exchange it with new fluid appear a complicated task, even if with high potential in the high through output setting.

\section{SNAP LB APA Pores}

APA SNAP microarray coupled with LB nanotemplate and cell free expression system (Figure 3), is being used as a platform for crystallizing proteins being expressed by the Pure-Express cell-free system produced by New England Biolab [19,20]. APA microarray will utilize as protein expressing system [20] for nanobiocrystallography based on LangmuirBlodgett nanotemplate, where APA channels/pores will be used as very small crystallization wells for proteins and precipitate solution where the overall system will be immersed. Nanoarray APA technology in combination with SNAP Genes technology, based on bacterial cell free expression system [18], appear to form a single approach capable of

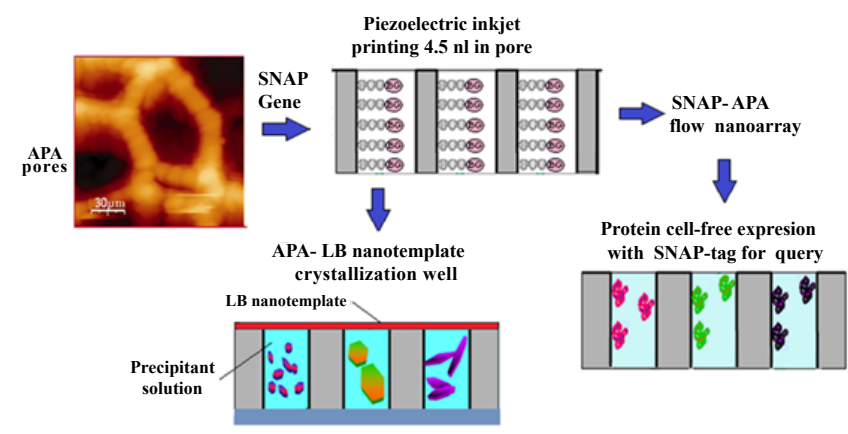

Figura 3: Figura 3 APA Microarrays utilizing piezoelectric inkjet and APA LB for nanocrystallography. A SNAP-APA flow application is also shown in Reference 19. 
effectively contributing to the solution of the numerous problems still present in protein nanocrystallography. This approach, by piezoelectric inkjet printing $4.5 \mathrm{nl}$ of SNAP Genes directly into each pore on the array, represents the future promising development of APA (Figure 3 ) in the area of protein crystallization now successfully in progress with LB lysozyme nanotemplate and cell-free expression of SNAP lysozyme genes immobilized in each APA micropores [20]. On the same Figure 3 it is shown also a label-free detection of proteins and protein-protein interactions via Quartz-crystal microbalance with Dissipation monitoring (QCM_D) under flow conditions recently reported using NAPPA SNAP microarrays [21] and patented for vaccinology screening and design [20]. SNAP tag is a $20 \mathrm{kDa}$ mutant of the DNA repair protein O6-alkylguanine-DNA alkyl transferase that reacts specifically and rapidly with Benzylguanine (BG) derivatives (SNAP ligand), leading to irreversible covalent binding of the SNAP tag [21]. The genes encoding proteins of interest are deposited via Functionalization on the bottom of the nanoarray and expressed after adding the appropriate biochemical reagents. Once translated, the gene produces the protein of interest and in the APA pore using a LangmuirBlodgett and adding the reagents and solvents for crystallization we get the same crystal of the protein of interest. The advantages with respect to existing technique are: 1 ) small volumes of analytes; 2 ) high sensitivity and specificity; 3) undenatured proteins in their natural environment, avoiding subsequent purification and expensive and wasteful passages; 4) effective protein crystallization including membrane, currently under-represented in PDB; 5) implementation of screening of vaccine

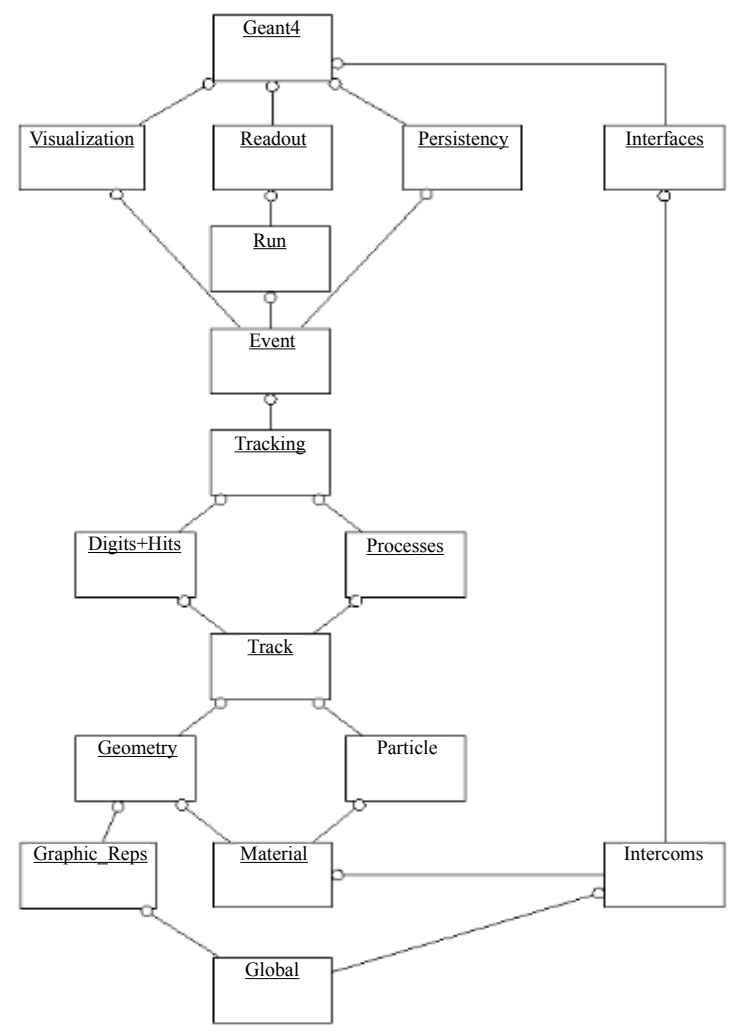

Figure 4: Flow Chart of GEANT4 from CERN (GEometric and Tracking), a Monte Carlo Simulation in $\mathrm{C}++$ of the passage of particles into matter that is being presently adapted to synchrotron interaction with protein crystals. immunogenicity, offering a useful platform to design and development of new vaccine preparations.

\section{Montecarlo Simulation}

Monte Carlo simulations have been typically and extensively used on high energy nuclear physics correct and interpret experimental data $[22,23]$. These systems were efficient and reliable tools for the study of low cross section reactions involving proton-antiproton annihilations in order to investigate the formation of high mass $(>2$ $\mathrm{GeV}$ ) bosons which decay into pure gamma $[22,23]$. Are exactly these very same Monte Carlo simulations that once properly adapted should be utilized in our opinion to evaluate the dose delivered to a crystal sample during planned experiments of nanocrystallography before the usage of synchrotron radiation. In Figure 4 is shown the flowchart of a typical Monte Carlo simulation that helps to describe geometry and material properties. Using this Program our Monte Carlo simulation is in progress (Bozdaganyan, Belmonte, Pechkova and Nicolini) to simulate the passage of $\mathrm{X}$-ray sychroton radiation into biological matter as protein crystal and since it is entirely written is $\mathrm{C}++$ allows easy programming. Our recently implemented Molecular Dynamics [25] by using the Newton's principles for the interactions of molecules permits to evaluate the interactions of secondary electrons, produced by photoelectron effects, with the structure of the sample crystal proteins. These simulations are being carried out using the actual geometry and the X-ray beam user-defined characteristics of the synchrotron facility beam (i.e. focus dimensions, energy/wavelength, and brilliance) with a phantom target. In our work in progress a homogeneous cube of material (i.e., thaumatin) is assumed to evaluate and compare absorbed dose with previously acquired experimental data. In a second phase of simulation this phantom will be substituted by a virtual crystal built ad-hoc for a particular protein. Monte Carlo (MC) estimation of radiation damage induced by high Energy X-ray Beam Interacting on the Protein Crystals will be completed before the actual usage of the synchrotron facility, while today dose can be calculated only after that crystals are damaged! User in this case will construct structures of Protein crystals using Geant4 [24] and will compare MC simulation of LB vs Classical crystals basing on what we have determined down to the atomic scale by all previously described experiments [1-21]. In this context are worthy of citation the references which describe the closest state of the art published by other groups of the world quite active in the key technologies here described such as cell free expression [26,27], Nucleic Acid Programmable Protein Arrays fluorescently labeled [28,29], Protein production [30] and Anodic Porous Allumina [31].

\section{References}

1. Nicolini C (2008) Nanobiotechnology and Nanobiosciences, Pan Stanford Series on Nanobiotechnology Volume 1 pp. 118-147. (ISBN 978-981-4241-380 ) London-New York-Singapore.

2. Pechkova E, Nicolini C (2002) From art to science in protein crystallization by means of thin film technology. Nanotechnology 13: 460-464.

3. Pechkova E, Nicolini C (2004) Protein nanocrystallography: a new approach to structural proteomics. Trends Biotechnol 22: 117-122.

4. Pechkova E, Zanotti G, Nicolini C (2003) Three-dimensional atomic structure of a catalytic subunit mutant of human protein kinase CK2. Acta Crystallogr D Bio Crystallogr 59: 2133-2139.

5. Nicolini C, Pechkova E (2006) Structure and growth of ultrasmall protein microcrystals by synchrotron radiation: I. microGISAXS and microdiffraction of P450scc. J Cell Biochem 97: 544-552.

6. Pechkova E, Vasile F, Spera R, Nicolini C (2008) Crystallization of alpha and beta subunits of IF2 translation initiation factor from Archabacteria Sulfolobus Solfataricus. Journal of Crystal Growth 310: 3767-3770. 
Citation: Nicolini C, Pechkova E (2014) New Trends in Protein Nanocrystallography Based On LB Nanotemplate, Cell Free Expression, SNAP APA and Montecarlo: A Review. J Microb Biochem Technol 6: 366-369. doi:10.4172/1948-5948.1000170

7. Pechkova E, Tripathi S, Spera R, Nicolini C (2008) Groel crystal growth and characterization. Biosystems 94: 223-227.

8. Pechkova E, Scudieri D, Belmonte L, Nicolini C (2012) Oxygen-bound Hell's gate globin I by classical versus LB nanotemplate method. J Cell Biochem 113 2543-2548.

9. Pechkova E, Tripathi S, Ravelli RB, McSweeney S, Nicolini C (2009) Radiation stability of proteinase $\mathrm{K}$ crystals grown by LB nanotemplate method. J Struct Biol 168: 409-418.

10. Gebhardt R, Pechkova E, Riekel C, Nicolini C (2010) In situ muGISAXS: I. Experimental setup for submicron study of protein nucleation and growth. Biophys J 99: 1256-1261.

11. Gebhardt R, Pechkova E, Riekel C, Nicolini C (2010) In situ muGISAXS: II. Thaumatin crystal growth kinetic. Biophys J 99: 1262-1267.

12. Nicolini C, Belmonte L, Maksimov G, Brazhe N, Pechkova E (2013) In situ Monitoring By Raman Spectroscopy of Lysozyme Conformation during "Nanotemplate" Induced Crystallization. J Microb Biochem Technol 6: 009-016.

13. Pechkova E, Sivozhelezov V, Belmonte L, Nicolini C (2012) Unique water distribution of Langmuir-Blodgett versus classical crystals. J Struct Biol 180: 57-64.

14. Pechkova E, Tropiano G, Riekel C, Nicolini C (2004) Radiation stability of protein crystals grown by nanostructured templates: synchrotron microfocus analysis. Spectrochimica Acta B 59: 1687-1693.

15. Pechkova E, Sartore M, Giacomelli L, Nicolini C (2007) Atomic force microscopy of protein films and crystals. Rev Sci Instrum 78: 093704.

16. Pechkova E, Belmonte L, Riekel C, Popov D, Koenig C, et al. (2013) LaserMicrodissection of Protein Crystals Down to Submicron Dimensions. J Nanomed Nanotechol S15: 002

17. Nicolini C, Correia TB, Stura E, Larosa C, Spera R, et al. (2013) Atomic Force Microscopy and Anodic Porous Allumina of Nucleic Acid Programmable Protein Arrays. Recent Pat Biotechnol 7: 111-121.

18. Pechkova E, Chong S, Tripathi S, Nicolini C (2010) Cell free expression and APA for NAPPA and protein crystallography, in Functional Proteomics and Nanotechnology-based Microarrays, (Eds. Nicolini, LaBaer) Pan Stanford Series on Nanobiotechnology Vol 2, Ch 7, pp. 121-147, London-New YorkSingapore.
19. Nicolini C (2013) Mass Spectrometry and Florescence Analysis of Snap-Nappa Arrays Expressed Using E. coli Cell_Free Expression System. J Nanomed Nanotechnol 4: 181

20. Nicolini C, Nicola B, Evgenyia P (2013) APA-SNAP Nanoarrays for LB Proteomics and Vaccinology, UIBM GE2012A000080 2013.

21. Nicolini C, Bragazzi N, Pechkova E (2012) Nanoproteomics enabling personalized nanomedicine. Adv Drug Deliv Rev 64: 1522-1531.

22. DeMarzo C, Guerriero L, Nicolini C, Posa F, Vaccai E, et al. (1975) Differential cross sections for backward $\pi-p \rightarrow n \pi 0$ from 2.6 to $8 \mathrm{GeV} / \mathrm{c}$. Physics Letters 56: 487-490.

23. DeMarzo C, Distante A, Guerriero L, Nicolin C, Posa F, et al. (1975) A 4T scintillation counter-optical spark chamber system for neutral particles. Nuc Instr Meth 131: 47-60.

24. Lamanna E, FiorilloA, Gallo A, Belmonte L (2010) Dosimetric Study in Human Head for CT Investigation of Inner Ear Using the Geant4 Toolkit, 2010 IEEE Nucl Science Symp Conf 1839-1842.

25. Bozdaganyan M, Pechkova E, Bragazzi NL, Nicolini C (2014) LB Identification of best crystallization methods by molecular dynamics, CREGE 311-324.

26. Spirin AS, Swartz JR (2008) Cell-free protein synthesis: methods and protocols. John Wiley \& Sons.

27. Smith MT, Berkheimer SD, Werner CJ, Bundy BC (2014) Lyophilized Escherichia coli-based cell-free systems for robust, high-density, long-term storage. Biotechniques 56: 186-193.

28. Ramachandran N, Hainsworth E, Bhullar B, Eisenstein S, Rosen B, et al (2004) Self-assembling protein microarrays. Science 305: 86-90.

29. Ramachandran N, Raphael JV, Hainsworth E, Demirkan G, Fuentes MG, et al. (2008) Next-generation high-density self-assembling functional protein arrays. Nature Methods 5: $535-538$.

30. Structural Genomics Consortium; China Structural Genomics Consortium Northeast Structural Genomics Consortium, Gräslund S, Nordlund P, Weigelt J, Hallberg BM, et al. (2008) Protein production and purification. Nat Methods 5: $135-146$.

31. Brüggemann D (2013) Nanoporous Aluminium Oxide Membranes as Cell Interfaces. Journal of Nanomaterials 18 pages. 